\title{
Projecto de Instalações Eléctricas Secção Técnica Vs Secção Económica de Canalizações Eléctricas
}

\section{Introdução}

O projecto das instalações eléctricas deve responder a critérios de ordem técnica, nomeadamente no que se refere à garantia da protecção das pessoas e instalações, mas contrapõem-se necessariamente os aspectos de ordem económica; resultará do compromisso entre estas duas posições contrastantes a definição daquela que será a solução mais acertada para uma dada instalação.

No capítulo dos custos associados a uma instalação eléctrica tem um peso crucial a energia desperdiçada durante o funcionamento da mesma, duração esta que pode em média considerar-se compreendida entre 20 e 30 anos.

Este desperdício tem duas origens: perdas excessivas por ineficiente concepção das instalações e selecção não criteriosa de equipamentos que utilizam a energia eléctrica e malbaratamento da energia eléctrica por funcionamento além do necessário.

Põe-se, portanto, também neste domínio a questão da eficiência energética.

Assim, o responsável pela concepção de uma instalação eléctrica deverá procurar não somente a solução técnica funcional da mesma mas preocupar-se que essa solução seja igualmente eficiente do ponto de vista energético.
A abordagem dum projecto eléctrico eficiente sob o ponto de vista energético deverá contemplar os seguintes pontos:

a) Minimização de perdas no sistema de distribuição

b) Redução das perdas devido ao desperdício na utilização do equipamento eléctrico

c) Redução das perdas associadas aos problemas associados à qualidade da energia

d) Prever as instalações para incorporarem aparelhagem de contagem e medida para fins de monitorização e de realização de auditorias eléctricas

\section{A Secção Técnica dos Condutores}

A definição técnica de canalizações em instalações de utilização de energia eléctrica, deve ser realizada de acordo com o definido nas Regras Técnicas de Instalações Eléctricas de Baixa Tensão, e assenta na verificação das seguintes condições:

- Critério do Aquecimento;

- Critério da protecção contra sobreintensidades

\section{A Secção Económica dos Condutores Calculada a Partir da Norma CEI/IEC60287-3-2}

Os métodos de cálculo económico dos condutores levam em linha de conta não somente o custo inicial dos mesmos e da sua instalação mas também os custos associados à exploração, isto é, os custos das perdas por efeito Joule.

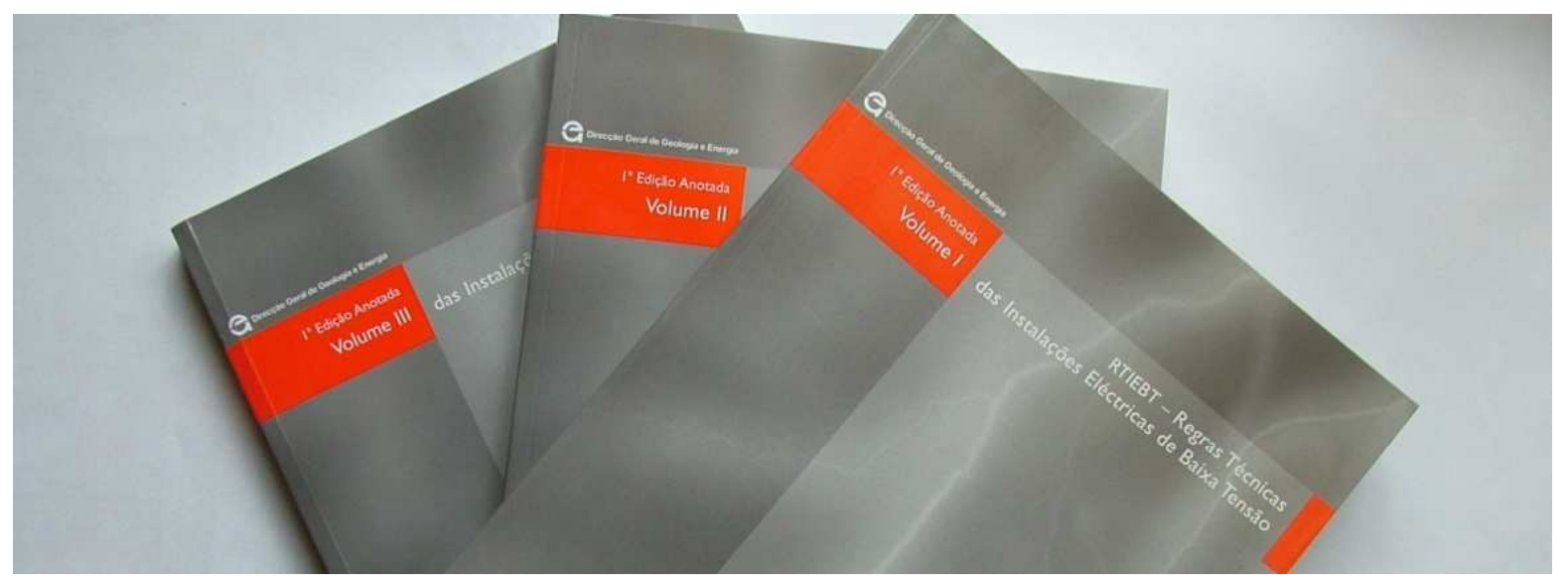




\section{ARTIGO TÉCNICO}

A norma CEI/IEC 60 287-3-2 - Electric cables - Calculation of the current rating -Economic optimization of power cable size (CEI, 1995) apresenta duas metodologias de cálculo uma baseada na determinação de gamas económicas de corrente, para diferentes cabos empregados, e uma outra, conhecida a corrente de projecto, que determina a secção que minimiza a função custo total.

$\mathrm{CT}=\mathrm{Cl}+\mathrm{CE}$

Onde:

CT - custo total

$\mathrm{Cl}$ - custo de investimento

CE - custo de exploração

Perda de energia no 10 ano:

$\mathrm{E}=\left(I_{\max }^{2} \times R \times L \times N_{p} \times N_{c}\right) T$

Onde:

Imax - corrente de pico do diagrama

$\mathrm{R}$ - resistência CA por unidade de comprimento

L - comprimento da canalização

$\mathrm{Np}-\mathrm{n}$ o de condutores sob idênticas condições

$\mathrm{Nc}-\mathrm{n}$ o de circuitos idênticos

T - no de horas de utilização das perdas

A resistência unitária $R$ é definida através do seu valor em CC e leva em consideração quer os efeitos pelicular e de proximidade $-y_{S}$ e $y_{p}$, quer as perdas em ecrãs metálicos e armaduras $-\lambda_{1}$ e $\lambda_{2}$.

Em virtude da variação da corrente, factor de carga $\neq 1$, e da possibilidade de incremento da mesma, a temperatura do condutor será diferente da correspondente à corrente máxima admissível $\theta(\theta z)$.
A norma considera uma temperatura média igual a:

$\theta_{m}=\frac{\theta-\theta_{a}}{3}+\theta_{a}$

Onde:

$\theta=$ temperatura correspondente $\mathrm{a} I z$

$\theta a=$ temperatura ambiente

$\mathrm{T}=\mathrm{n}$ ㅇ de horas de utilização das perdas

O número de horas de utilização das perdas é dado pela relação:

$T=\int_{0}^{8760} \frac{I^{2}(t)}{I_{\mathrm{máx}}^{2}} d t$

Na Fig. 1 podemos apreciar um diagrama de carga diário, que traduz a variação da corrente com o tempo.

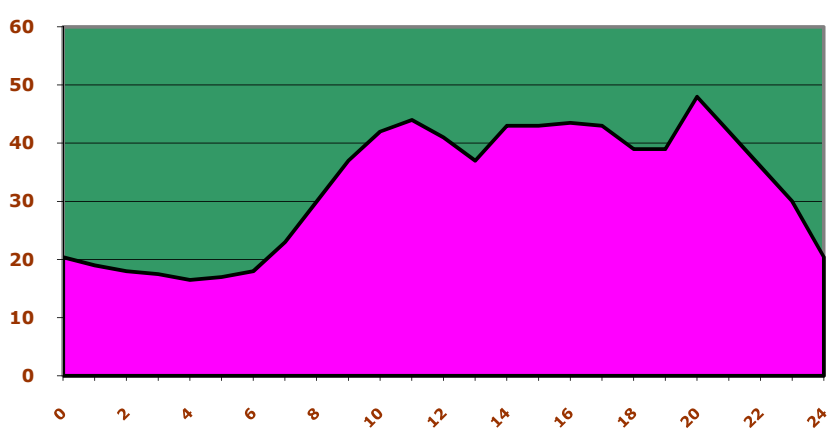

Figura 1 - Diagrama de carga I(t)

A Fig. 2 representa o chamado diagrama normalizado $\pi(t)$ que resulta do precedente dividindo-o por $I_{\text {máx }}$ A ordenada máxima passa a ser obviamente 1 .

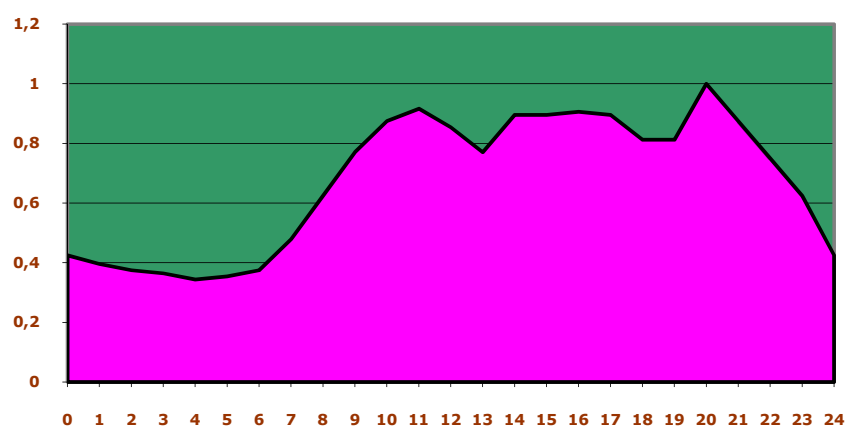

Figura 2 - Diagrama normalizado $\pi(t)$ 
A Fig. 3 corresponde ao diagrama $\pi^{2}(t)$.

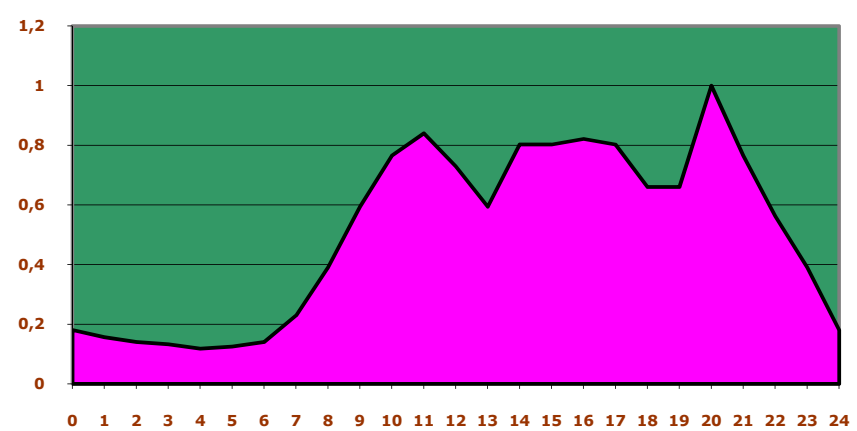

Figura 3 - Diagrama normalizado $\pi^{2}(t)$

Como se trata da elevação ao quadrado de valores quando muito iguais a 1 virá mais cavado.

$T=\int_{0}^{8760} \pi^{2}(t) d t$

O no de horas de utilização das perdas é referido ao período de 8760 h (1 ano). A estimação deste valor vem normalmente dada através da fórmula:

$T=\mu \times 8760$

$\mu=p \times f_{c}+(1-p) \times f_{c}^{2}$

Onde:

$\mu=$ factor de carga das perdas

$\mathrm{fc}=$ factor de carga

$p=$ coeficiente

(igual a 0,3 - redes de transporte, e igual a 0,2 - redes de distribuição (IEEE, 1990)

Custo das perdas no 10 ano:

$C E_{E}=\left(I_{\max }^{2} \times R \times L \times N_{p} \times N_{c}\right) T \times P$

Onde:

$\mathrm{CE}_{\mathrm{E}}=$ custo de exploração por perda de energia no 1ㅇa ano $\mathrm{P}=$ custo do $\mathrm{Wh}$
Custo da potência de perdas:

$$
C E_{p}=\left(I_{\max }^{2} \times R \times L \times N_{p} \times N_{c}\right) \times D
$$

Onde:

$\mathrm{CE}_{\mathrm{p}}=$ custo de exploração por solicitação de potência adicional

$\mathrm{D}=$ custo anual da potência

Custos de exploração (1ㅇano):

$$
C E=\left(I_{\max }^{2} \times R \times L \times N_{p} \times N_{c}\right)(T P+D)
$$

Considerando-se os pagamentos feitos no fim do ano, os custos no iésimo ano virão dados por:

$$
C E_{i}=C E\left[(1+a)^{2}(1+b)\right]^{i-1}
$$

Admitindo-se crescimento percentual anual da carga de $a$ e dos custos de energia e potência de $b$.

Há duas abordagens para lidar com pagamentos feitos em tempos diferentes, o investimento no início da exploração da instalação e os custos durante o tempo de vida da mesma:

- método das anuidades ;

- $\quad$ método da actualização.

O método da actualização é mais geral e é o usado na norma.

$$
\begin{aligned}
& r=\frac{(1+a)^{2}(1+b)}{(1+i)} \\
& Q=\frac{1-r^{N}}{1-r}
\end{aligned}
$$

Onde:

i = taxa de actualização

$\mathrm{N}=\mathrm{n}$ ㅇ de anos de vida média da instalação 


\section{ARTIGO TÉCNICO}

$$
C E_{a}=\left(I_{\max }^{2} \times R \times L \times N_{p} \times N_{c}\right)(T P+D) \times \frac{Q}{1+i}
$$

Onde:

$\mathrm{CEa}=$ custo de $\mathrm{N}$ anos de exploração da instalação, referidos ao início do empreendimento, isto é, actualizados.

Seja F uma variável auxiliar dada por:

$F=N_{p} \times N_{c}(T P+D) \times \frac{Q}{1+i}$

A função custo total virá então com a forma:

$$
\begin{aligned}
& C T=C I+I_{\text {máx }}^{2} R L F \\
& R=\frac{\rho_{20} \times B\left[1+\alpha_{20}\left(\theta_{m}-20\right)\right]}{S} \times 10^{6} \Omega / \mathrm{m} \\
& B=\left(1+y_{s}+y_{p}\right)\left(1+\lambda_{1}+\lambda_{2}\right)
\end{aligned}
$$

Onde:

$\rho 20=$ resistividade do condutor a $20 \circ \mathrm{C}$ em CC

$\theta \mathrm{m}=$ temperatura média

Admitindo um custo de investimento dado por:

$$
C I=(A \times S+C) \times L
$$

Onde:

$A=$ termo dependente da secção do cabo

$\mathrm{C}=$ termo constante

A secção económica virá dada pela fórmula:

$S_{e c}=1000 \times I_{\text {máx }} \sqrt{\frac{F \times \rho_{20} \times B\left[1+\alpha_{20}\left(\theta_{m}-20\right)\right]}{A}} \mathrm{~mm}^{2}(20)$ A secção económica será normalizada para o valor comercial mais próximo.

\section{Método de fixação dos valores máximos de perdas admissíveis nas canalizações}

Este método é preconizado pela Região Administrativa de Hong-Kong, que através do Departamento Eléctrico e Mecânico do Governo (EMSD) definiu uma abordagem no domínio da baixa tensão, até 2008 de carácter voluntário, mas que a partir de 2009 será integrado na legislação, tornando-se, por conseguinte de carácter obrigatório (EMSD, 1997).

A metodologia sugerida pelo presente método conduziu a resultados significativos no que se refere à poupança de energia eléctrica, comprovados pelo EMSD, no decorrer de estudos realizados nos últimos 30 anos, junto dos operadores que voluntariamente adoptaram este procedimento (EMSD, 1997), (Hui, 2003).

A metodologia consiste em fixar os valores máximos de perdas admissíveis nas canalizações, ou seja, a de impor rendimentos mínimos das linhas.

Para além das condições técnicas de temperatura e queda de tensão a máxima perda de potência passa a ser critério de dimensionamento.

Consideram-se duas situações:

- circuitos trifásicos lineares equilibrados;

- $\quad$ trifásicos não-lineares.

\section{0 caso - Circuito trifásico linear equilibrado}

Potência transportada e perdas nos condutores do circuito:

$$
P=\sqrt{3} \times U_{c} \times I_{b} \times \cos \varphi
$$

$$
p=3 \times I_{b}^{2} \times r \times L
$$

Onde:

$\mathrm{lb}=$ corrente do circuito 
$\mathrm{L}=$ comprimento dos condutores

$r$ = resistência em CA por metro de canalização à

temperatura de funcionamento

O valor percentual $p_{r}$ das perdas vem dado pela relação:

$p_{r}=\frac{3 \times I_{b}^{2} \times r \times L}{\sqrt{3} \times U_{c} \times I_{b} \times \cos \varphi}$

Donde, definido o valor percentual que se admite para as perdas, se determinará o máximo valor para a resistência $r$.

$r_{\text {máx }}=\frac{p_{r} \times U_{c} \times \cos \varphi \times 1000}{\sqrt{3} \times I_{b} \times L} \quad \mathrm{~m} \Omega / \mathrm{m}$

Encontrado o valor de $r_{\text {máx }}$ as tabelas fornecerão a secção a utilizar.

Correcção das perdas nos cabos devido às diferentes temperaturas de funcionamento.

A temperatura do condutor pode ser aproximada através da seguinte fórmula:

$\theta_{c}=\theta_{a}+\frac{I_{b}^{2}}{I_{z T}^{2}}\left(\theta_{z}-30\right)$

Onde:

$\theta_{c}=$ temperatura do condutor

$\theta_{\mathrm{a}}=$ temperatura ambiente

$\mathrm{I}_{\mathrm{ZT}}=$ corrente máxima admissível no cabo dada pelas tabelas

$\theta_{z}=$ temperatura máxima admissível nos condutores

A resistência à temperatura $\theta c$ pode ser expressa a partir da relação:

$R_{c}=R_{0}\left(1+\alpha_{0} \times \theta_{c}\right)$

Onde:

$\mathrm{R}_{0}=$ resistência do condutor a 0 ㅇ C $\alpha_{0}=$ coeficiente de termorresistividade a 0 으

Donde:

$\frac{R_{c}}{R_{z}}=\frac{1+\alpha_{0} \times \theta_{c}}{1+\alpha_{0} \times \theta_{z}}=\frac{234,4+\theta_{c}}{234,4+\theta_{z}}$

20 caso - Circuito trifásico não-linear, equilibrado, com valores conhecidos de corrente lb e de taxa de distorção harmónica THD

A potência aparente transportada pelo circuito será dada por:

$S=\sqrt{3} \times U_{c} \times I_{b}$

Onde:

$I_{b}=\sqrt{\sum_{h=1}^{\infty} I_{h}^{2}}=\sqrt{I_{1}^{2}+I_{2}^{2}+I_{3}^{2} \ldots}$

$T H D=\frac{\sqrt{\sum_{h=2}^{\infty} I_{h}^{2}}}{I_{1}}$

$$
I_{b}=I_{1} \sqrt{1+T H D^{2}}
$$

$I_{1}=\frac{I_{b}}{\sqrt{1+T H D^{2}}}$

Admitindo baixa distorção da tensão, o que é razoável, a potência activa transportada terá por expressão:

$P=\sqrt{3} \times U_{c} \times I_{1} \times \cos \varphi$

Onde:

11 = componente fundamental da corrente

$\cos \varphi=$ factor de potência do circuito 


\section{ARTIGO TÉCNICO}

Desprezando os efeitos pelicular e de proximidade, as perdas nos condutores, incluindo o condutor neutro, será:

$p=\left(3 \times I_{b}^{2}+m \times I_{N}^{2}\right) \times r \times L$

$I_{N}=3 \times \sqrt{I_{3}^{2}+I_{6}^{2}+I_{9}^{2} \cdots}$

$m=\frac{S_{F}}{S_{N}}$

Que é a relação entre as secções de fase e de neutro.

As perdas percentuais (pr) na canalização virão então dadas por:

$p_{r}=\frac{\left(3 \times I_{b}^{2}+m \times I_{N}^{2}\right) \times r \times L}{\sqrt{3} \times U_{c} \times I_{1} \times \cos \varphi}$

E a máxima resistência unitária a atribuir ao cabo:

$r_{\text {máx }}=\frac{p_{r} \times \sqrt{3} \times U_{c} \times I_{1} \times \cos \varphi \times 1000}{\left(3 \times I_{b}^{2}+m \times I_{N}^{2}\right) \times L}$

As tabelas especificarão a secção do cabo a considerar.

A correcção de temperatura poderá ser feita mediante a fórmula aproximada seguinte:

$\theta_{c}=\theta_{a}+\frac{\left(3 \times I_{b}+m \times I_{N}\right)^{2}}{\left(3 \times I_{z T}\right)^{2}}\left(\theta_{z}-30\right)$

Alguns valores de referência para $p_{r}$ :

Tabela I - Valores máximos das perdas percentuais para diferentes tipos de circuitos

\begin{tabular}{|l|c|}
\hline \multicolumn{1}{|c|}{ Tipo de circuito } & Pr \\
\hline $\begin{array}{l}\text { Ligações entre transformadores de distribuição } \\
\text { e Quadros Gerais }\end{array}$ & $\begin{array}{c}\text { Máx. } \\
0,5 \%\end{array}$ \\
\hline $\begin{array}{l}\text { Canalizações entre Quadros Gerais e Quadros } \\
\text { Parciais }\end{array}$ & $1,5 \%$ \\
\hline $\begin{array}{l}\text { Circuitos terminais: iluminação, tomadas ou } \\
\text { outros usos com correntes acima de 32 A }\end{array}$ & $1 \%$ \\
\hline $\begin{array}{l}\text { Colunas montantes e entradas, (até 2,5\% para } \\
\text { utilizações domésticas) }\end{array}$ & $1,5 \%$ \\
\hline $\begin{array}{l}\text { Alimentações de grandes cargas como motores } \\
\text { de potência apreciável }\end{array}$ & $2,5 \%$ \\
\hline
\end{tabular}

\section{Conclusões}

A busca da eficiência e da utilização racional de energia (URE), particularmente nos sistemas eléctricos, leva a considerar todos aqueles aspectos que concorrem para realizar esse fim. A consideração do rendimento das canalizações, já contemplada nas áreas do transporte e grande distribuição, faz todo o sentido aplicada às redes de baixa tensão, até pela sua enorme extensão.

Em Hong-Kong, onde o sistema de fixação de perdas máximas se encontra implementado há alguns anos, o dimensionamento económico de condutores insere-se num programa mais vasto de URE em edifícios de serviços, contemplando vertentes tais como instalações de iluminação, sistemas de aquecimento, ventilação e ar condicionado, transporte por elevadores, monta-cargas e escadas rolantes e o desempenho energético de edifícios apresentando resultados tangíveis significativos.

\section{Fontes de Informação Relevantes}

[CEI, 1995] CEI IEC 60 287-3-2, Electric cables, Calculation of the current rating - Part 3 - Section 2, Suiça, 1995.

[Cooper, 1997] Copper Development Association, Electrical Energy Efficiency, U.K., 1997.

[EMSD, 1997] Electrical and Mechanical Services Department (EMSD), Code of Practice for Energy Efficiency of Electrical Installations, Hong-Kong, 2005.

[Anders, 1997] Anders J George, Rating of Electric Power Cables, McGraw-Hill, Nova lorque, 1997.

[Hui, 2003] Hui, Sam C. M., Energy Efficiency and Environmental Assessment for Buildings in Hong Kong, MECM LEO Seminar, Advances on Energy Efficiency and Sustainability in Buildings, pag, 21-22, 2003, Kuala Lumpur, Malaysia.

[Silva, 2009] Silva H.J., Gomes A.A., Ramos S.C., “A definição do valor máximo das perdas nas canalizações eléctricas como medida de eficiência energética", JLBE09 - Jornadas LusoBrasileiras de Ensino e Tecnologia em Engenharia 2009, 10 a 13 de Fevereiro de 2009, Instituto Superior de Engenharia do Porto, Porto, Portugal. 